\title{
Lower Body Realization of the Baby Humanoid - 'iCub'
}

\author{
N.G.Tsagarakis \\ Italian Institute of Technology \\ Genova, Italy \\ nikos.tsagarakis@iit.it
}

\author{
F. Becchi \\ TELEROBOT \\ Genova, Italy \\ francesco.becchi@telerobot.it
}

\author{
L.Righetti \\ BIRG, EPFL \\ Lausanne, Switzerland \\ ludovic.righetti@a3.epfl.ch
}

\author{
A.J. Ijspeert \\ BIRG, EPFL \\ Lausanne, Switzerland \\ auke.ijspeert@epfl.ch
}

\author{
D.G.Caldwell \\ Italian Institute of Technology \\ Genova, Italy \\ darwin.caldwell@iit.it
}

\begin{abstract}
Nowadays, the understanding of the human cognition and it application to robotic systems forms a great challenge of research. The iCub is a robotic platform that was developed within the RobotCub European project to provide the cognition research community with an open babyhumanoid platform for understanding and development of cognitive systems.

In this paper we present the design requirements and mechanical realization of the lower body developed for the "iCub". In particular the leg and the waist mechanisms adopted for lower body to match the size and physical abilities of a $2 \frac{1}{2}$ year old human baby are introduced.
\end{abstract}

\section{INTRODUCTION}

During the last decade the rapidly growing emergence of humanoid robots was mainly due their potential observed within a number of intrinsic features of these robotic systems. Specific inherent features of this kind of platforms include but not limited to anthropomorphism, friendly design, natural and adaptive locomotion and human friendly behavior. These features make humanoid robots very suitable for prospective applications in all kinds of human environments and daily activities. As a result, multi degree of freedom human like robots have become very common. Following the development of the first humanoid back in 1973 [1] a number of other humanoid platforms in different sizes were developed. The large scale anthropomorphic prototypes include the H6, H7 [2], P2 [3], ASIMO [4], JOHNNIE and LOLA [5], WABIAN-2 [6] KHR-2 [7] and LUCY [8], while the HRP, HRP-2 [9], SAIKA [10] and KENTA [11] are examples of medium size humanoid robots. In addition to the above examples of adult or medium sized anthropomorphic robots a number of smaller prototypes have been also constructed including MK.5 [12], PINO [13], the SDR-3 and SDR-4X [14] and the humanoidgorilla robot developed by [15].

The RobotCub project is a research initiative dedicated to the realization of embodied cognitive systems and the creation of an advanced robotic platform for neuroscientific study. The two main goals of this project are; i) Creation of an open hardware/software humanoid robotic platform for research in embodied cognition. This is the iCub.

ii) Advancing our neural understanding of cognitive systems by exploiting this platform in the study of the development of cognitive capabilities in humanoid robots.

The iCub platform has as its aim the replication of the physical and cognitive abilities of a $2 \frac{1}{2}$ year old baby. This "baby" robot will act in a cognitive scenario, performing the tasks useful to learning, interacting with the environment and humans [16].

The small $(90 \mathrm{~cm}$ tall), compact size $(<23 \mathrm{~kg}$ and fitting within the volume of a child) and high number (53) of degrees of freedom combined with the OPEN approach for research in cognitive development form fundamental differences with the many excellent humanoids already developed.

The OPEN approach (both for hardware and software) which is the open access to the research community of the software and hardware modules of the iCub will allow a wide range of experimentation both in the software but also at the hardware mechanical/sensory level by an enlarged user group speeding the development of the cognitive paradigms.

The development of such an OPEN structure that aims to match the functionality described above poses many significant and important challenges and requires careful design, co-ordination and integration of the various components that form the complete mechatronic structure. It is evident that the OPEN nature of the iCub platform induces high needs for robustness and easy maintenance.

This paper reports on the design of the lower body modules for the iCub. The paper is organized as follows: Section II gives the specifications of the lower body. Sections III and IV introduces the mechanical design concepts, the actuation and the sensing of the lower body. Section V presents the characteristic measures of the current design in terms of joint range of motion, and output torque. Finally, section VI addresses the conclusions. 


\section{LOWER BODY SPECIFICATIONS}

Among the most important questions to be addressed when considering the hardware design is the fundamental layout, to enable a natural, stable, robust actions found in the early years development of a child. The fundamental layout of the lower body of the iCub include the definition of the number of D.O.F required and their actual location as well as the actual size of the legs and lower torso.

The size of the iCub will approximate all the dimensions of a two and a half year old child. For the definition of the number of D.O.F for the legs and the waist modules several iCub simulation models were developed and analyzed. For the legs these simulations have indicated that for crawling, which is the primary locomotion requirement of the iCub platform, sitting and squatting a 5 D.O.F leg is adequate. It was decided though to incorporate an additional D.O.F at the ankle to support not only crawling but also standing (supported and unsupported) and walking. As different research groups may also interested in walking and balance research the incorporation of the additional D.O.F to support the ankle lateral motion was considered as an advantage for the OPEN nature of the system. Therefore, each leg consists of 6 D.O.F: that include 3 D.O.F at the hip, 1 D.O.F at the knee level and 2 D.O.F at the level of the ankle. Despite the fact that the foot rotation was observed in baby subjects to be active during crawling the twist rotation was not implemented at the foot as was found from simulations not necessary for executing crawling motion patterns.

In designing the $\mathrm{iCub}$ waist we considered specific criteria such as the manipulation workspace of the arms and the motion flexibility of the upper body but also the kinimatic requirements for performing effecting crawling locomotion. Taking into consideration also the OPEN nature and the compact size of the iCub platform it was evident that compactness, easy of maintenance and robustness were also critical issues for this module as were for the whole platform. The crawling simulation analysis revealed that for effective crawling locomotion a 2 D.O.F waist is adequate. The flexibility of the upper body motions is directly related to the flexibility of the waist structure. In the majority of the existing humanoids robots the waist module is usually implemented with a simple 2 D.O.F mechanism, however humanoids that trying to replicate the functionally of the human spine have also been developed [17]. Despite the fact that the latest approach is very attractive as it provides the greatest flexibility of motion, it suffers from high complexity and control difficulties. This is due to the large number of actuators and sensors. For the iCub a 3 D.O.F waist was considered as this implementation offers greater motion flexibility than that of the conventional 2 D.O.F waist mechanisms while at the same time the complexity is kept low in line with the requirements of the OPEN platform for robustness, easy maintenance and manufacturing. Based on above, the iCub waist provides pitch, roll and yaw motions in the upper body.

Regarding the range of motion of the individual joints since the $\mathrm{iCub}$ is a human-like robot and will perform tasks similar to those performed by a human, the range of motion of a "standard" human baby was used as a starting point for the selection of the movable range of each joint in the iCub.
Table I shows the range of motions specification for the joints of the lower body in comparison with the corresponding ranges found in a human baby.

TABLE I

RANGE OF MOTION OF THE LOWER BODY JOINTS.

\begin{tabular}{|c|c|c|}
\hline & Human & iCub \\
\hline LEG & \multicolumn{2}{|c|}{ Range of motion $\left({ }^{\circ}\right)$} \\
\hline Hip rotation & $-43.5,+45.5$ & $-91,+31$ \\
\hline Hip Abduction/Adduction & $-40,+45$ & $-31,+45$ \\
\hline Hip Flexion/Extension & $-147,+45$ & $-120,+45$ \\
\hline Knee & $0,+127.5$ & $0,+130$ \\
\hline Ankle Flexion/Extension & $-51.5,+34$ & $-60,+70$ \\
\hline Ankle Abduction/Adduction & $-44.5,+58$ & $-25,+25$ \\
\hline Ankle Twist & $-34,+36.5$ & Not Implemented \\
\hline WAIST & & $-90,+90$ \\
\hline Waist roll & $-35,+35$ & $-10,+90$ \\
\hline Waist pitch & $-30,+70$ & $-60,+60$ \\
\hline Waist yaw & $-40,+40$ & \\
\hline
\end{tabular}

The range of the waist joints has been extended to increase the manipulation workspace of the baby-like robot. In particular the range of the waist yaw and roll has been increased while the range of the pitch motion was modified to increase the upper body forward tilting in order to improve the workspace for the iCub's arms when performing manipulation tasks while the robot is in a sitting position. After studying simulations of the performance of basic crawling activities and transitions to baby-style sitting positions, the range of motion of the leg joints were also modified. Simulation studies have confirmed that the range of motions provided in the specification is sufficient to ensure the $\mathrm{iCub}$ can perform the basic exploratory and manipulation procedures required for the "child".

Having determined the desired kinematic structure and range of motion of the joints, it was possible to move to structural and design considerations derived from dynamic performance criteria based on the masses, and projected forces and torques. The initial targets for the masses of all the limbs and mechanical structures were set using a "real" child model of $23 \mathrm{Kg}$ which is at the top end of normal mass for a young ( $2 \frac{1}{2}$ year old) child. A detailed breakdown of body segment masses and their lengths is shown in Table II.

TABLE II

MASS AND SIZE OF THE MAIN BODY SEGMENTS.

\begin{tabular}{|l|l|l|}
\hline Body Section & Mass (Kg) & Length $(\mathrm{m})$ \\
\hline Upper Arm & 1.15 & 0.15 \\
\hline Forearm (including the hand & 1.25 & 0.13 \\
\hline Upper Leg (Thigh) & 1.5 & 0.17 \\
\hline Lower Leg (Shin) & 1.5 & 0.17 \\
\hline Ankle and Foot & 0.5 & 0.05 \\
\hline Upper Torso & 3.75 & 0.12 \\
\hline Lower Torso & 6.5 & 0.12 \\
\hline
\end{tabular}

Using these projections and the kinematic layout as a baseline, dynamic simulations were developed to determine 
static and dynamic performance requirements for all joints and to measure the torques/velocities that would be needed by the actuators in order to achieve correct crawling motion.

The simulations were done with Webots [18], a simulator based on ODE (Open Dynamic Engine) which is an open source library for simulating $3 \mathrm{D}$ rigid body dynamics. These simulations, Fig.1, particularly focused on performing crawling motions at different gait speeds $(0.5 \mathrm{~Hz}$ cycles and $1 \mathrm{~Hz}$ cycles) and transitions from sitting to crawling pose and vice versa.

The crawling motion consisted of simple sinusoidal trajectories that the joints had to follow using a simple PID controller. Typical torque curves for the major joints during $1 \mathrm{~Hz}$ crawling are shown in Fig. 2.
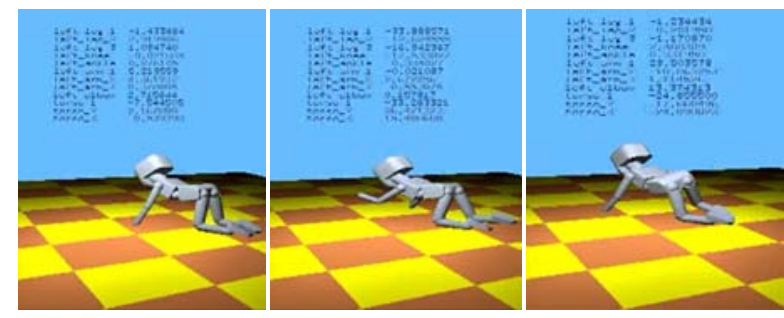

Fig. 1 View of the iCub crawling simulation.
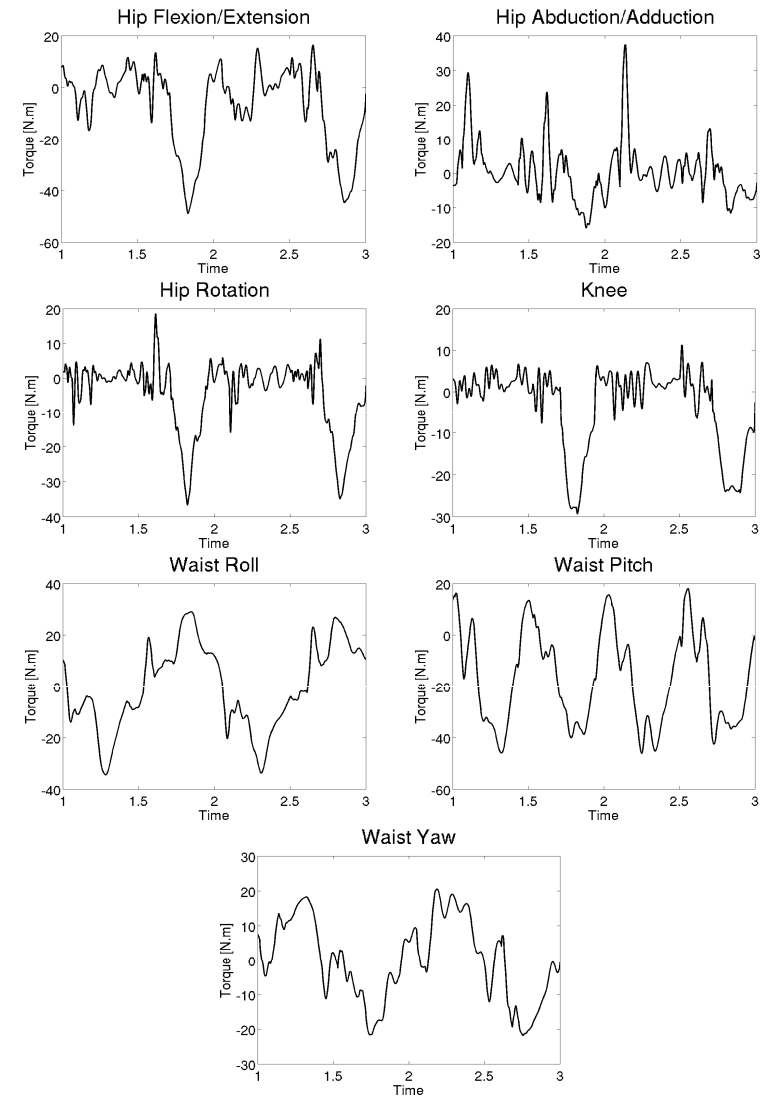

Fig. 2 Torque curves of the major joints of the iCub lower body during $1 \mathrm{~Hz}$ crawling simulation.

From these simulations, the maximum torque requirements of each joint were determined Table III.
TABLE III

PEAK TORQUES OF ICUB LOWER BODY JOINTS.

\begin{tabular}{|l|c|c|}
\hline JOINT & $\begin{array}{l}\text { Peak Torque } \\
(\mathrm{Nm}) \text { at } 0.5 \mathrm{~Hz}\end{array}$ & $\begin{array}{l}\text { Peak Torque } \\
(\mathrm{Nm}) \text { at } 1 \mathrm{~Hz}\end{array}$ \\
\hline Hip Flexion/Extension & 38.5 & 46.3 \\
\hline Hip Abduction/Adduction & 15.1 & 37.1 \\
\hline Hip Rotation & 23.2 & 36.8 \\
\hline Knee & 28.0 & 27.4 \\
\hline Ankle Flexion/Extension & 11.3 & 12.4 \\
\hline Waist Roll & 26.5 & 27.2 \\
\hline Waist Pitch & 34.3 & 45.8 \\
\hline Waist Yaw & 13.7 & 30.1 \\
\hline
\end{tabular}

\section{LOWER BODY MECHANICAL LAYOUT}

The mechanical realization of the lower body of the iCub baby humanoid robot and an overview of its kinematics with the location of the degrees of freedom is illustrated in Fig. 3 . The height of the iCub lower body from the foot to the waist is $611 \mathrm{~mm}$, the width of the lower torso from left to the right is $186 \mathrm{~mm}$ while the depth from front to back is $110 \mathrm{~mm}$. The weights of the first lower body prototype are as follows: the total weight is $12.7 \mathrm{Kg}$ with the weight of each leg being $3.5 \mathrm{Kg}$ and the weight of the lower torso including the waist being $5.7 \mathrm{Kg}$.


Fig. 3 Kinematic configuration and CAD model of iCub lower body.

To satisfy the size and weight requirements but also the high level of robustness required by the OPEN approach of the iCub the mechanical realization the lower body was based on the following two principles;

i) Modular structure

Since there is a strong belief that the platform will be replicated by a large number of research groups a modular structure will be beneficial for the manufacturing and maintenance process of the platform.

ii) Integrated actuator units

Based on their performance capabilities, three different actuator units are used to power the lower body. Motor gears, joint relative and absolute sensing have been highly integrated to optimize weight and dimensions of the lower body joints. 
From the kinematic point of view the iCub's lower body incorporates a differential waist and hip joint. In particular the pitch, yaw motions of the waist joint and the flexion and abduction of the hip joint are realized with a use of a cable differential mechanism providing increased stiffness which is an inherent feature of the differential mechanism.

The selection of the actuation of the individual joints of the iCub's lower body was based on the simulation data of the previous section in conjunction with the dimensional constraints imposed in trying to replicate the mechanical and kinematic functionality of a child. The actuation solution adopted for the $\mathrm{iCub}$ is based on a combination of a harmonic drive reduction system (CSG series, 100:1 ratio for all the major joints) and a brushless frameless motor (BLM) from the Kollmorgen frameless RBE series, Fig. 4.

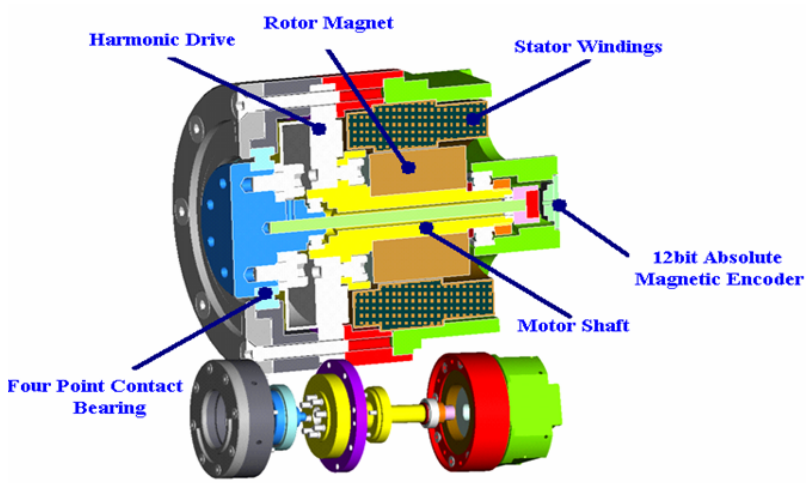

Fig. 4 View of the iCub motor/gearbox actuator group.

The harmonic drives include no-backlash, high reduction ratios on small space with low weight while the brushless motors exhibits desired properties such as robustness, higher power density, and higher torque and speed bandwidths when compared with conventional DC brushed motors. The use of frameless motors enables the high level of integration of the motor and harmonic system within an endoskeletal structure that minimises size, weight and dimensions with the immediate benefit of the freedom in shaping the actuator housing. To ease the fabrication and the maintenance process only three different actuators groups are used to power the major joints of the iCub.

i) the high power actuator group is capable of delivering over $40 \mathrm{Nm}$ at the output shaft and has a diameter of $60 \mathrm{~mm}$ and a length of $53 \mathrm{~mm}$,

ii) the medium power motor group provides up to $20 \mathrm{Nm}$ with a diameter of $50 \mathrm{~mm}$ and a length of $48 \mathrm{~mm}$, and

iii) the low power motor group delivers up to $11 \mathrm{Nm}$ with a diameter of $40 \mathrm{~mm}$ and a length of $82 \mathrm{~mm}$.

The adoption of the above harmonic/BLM actuator groups results in a robotic platform with limited inherent passive compliance. Although this compliant behaviour is vital in all kind of interactions between the robot and its environment the incorporation of such passive elastic elements was prevented by he lack if space in this small size humanoid robot. As an alternative, the mechanical elasticity in the $\mathrm{iCub}$ lower limb joints is implemented through active compliance control.

The components of the first prototype that are considered as low stressed parts were fabricated in
Aluminum alloy A16082 which is a structural alloy having a medium strength and excellent corrosion resistance. Medium/highly stressed parts components were made of Aluminum alloy 7075 (Ergal) that is one of the highest strength Aluminum alloys available. Its strength to weight ratio is excellent, and it is ideally used for medium and highly stressed parts such as actuator housing load bearing parts etc. Finally, the major joint shafts were fabricated from Stainless steel 17-4PH which delivers an excellent combination of good oxidation and corrosion resistance together with high strength.

\section{LOWER BODY MECHANICAL DETAILS}

\section{A. Waist Mechanism}

The iCub's waist was realized using a mechanism where the torque and power of the two actuators used for the upper body pitch and yaw motions is transferred to these two motions using a cable based differential mechanism as seen in Fig. 5.

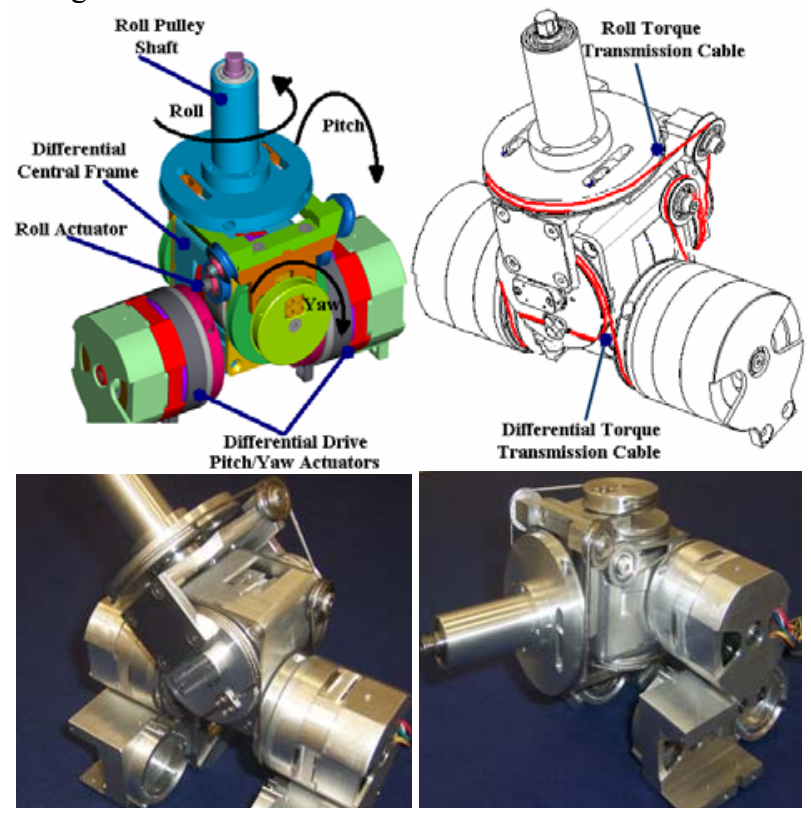

Fig. 5 The mechanical design of the differential 3 D.O.F iCub waist.

For the pitch motion of the waist the two high power actuators assemblies (40Nm each) that power the pitch and yaw motion apply a synchronous motion to the two directly coupled differential input wheels. For the yaw motion the motors turn in opposite directions and this generates the yaw action on the upper body. This differential mechanism has several advantages when compared with traditional serial mechanisms used in humanoids robots. These are;

i) Increased stiffness compared to serial waist mechanisms usually seen in most of the humanoids robots, this is inherent feature of the differential mechanism.

ii) The sum of the torque generated by the two actuators that power the differential joint can be distributed in both joints. 
iii) As a result of the previous feature smaller actuators can be used to achieve the maximum output torques required for the pitch and yaw motions.

The last is shown in Fig. 6 where solid lines shows the required motor torque curves for the differential pitch/yaw waist joint during $1 \mathrm{~Hz}$ crawling cycle while dash lines shows the equivalent torques for a waist joint which employs a serial mechanism.

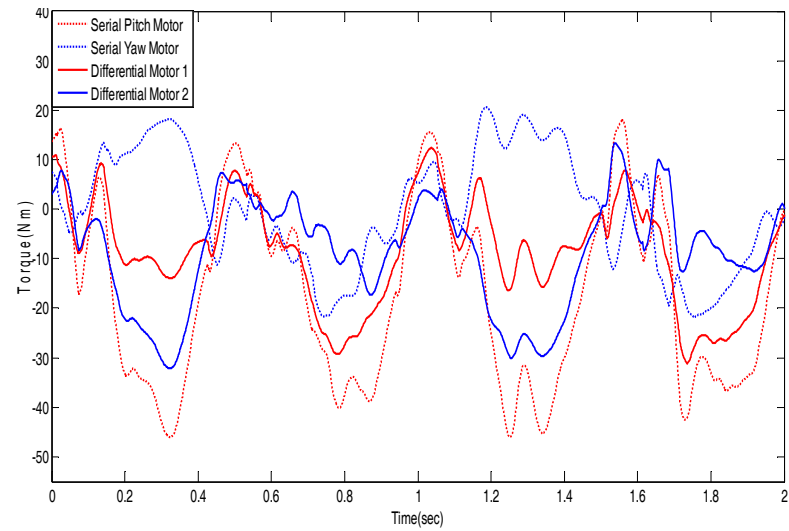

Fig. 6 Drive torque requirements comparison for a differential and a seria waist mechanism.

The roll motion is achieved through a pulley shaft that is directly connected to the upper body frame. The actuator assembly of the roll pulley $(20 \mathrm{Nm})$ is located within the square centre element of the differential, Fig. 5. The torque is conveyed through a cable transmission system that provides additional gearing (1.5:1) to meet the torque requirements of the roll joint, Table III.

\section{B. Leg Module}

For the leg design, particular attention was paid to satisfy the dimensional and weight requirements while at the same time maximizing the range of motion of each joint. The leg section has a modular structure that was designed for easy fitting/removal and maintenance. In general the leg has an anthropomorphic kinematic form consisting of the following major modules, the hip, the thigh with the knee joint, the calf with the ankle joint and the foot, Fig 7(a).

The hip module provides 2 D.O.F to enable the thigh flexion/extension, abduction/adduction. The realization of the first 2 D.O.F is also based on a cable differential mechanism similar to the one used in the waist. Two medium power actuator groups $(20 \mathrm{Nm})$ located in the lower torso are used to drive the two input pulleys of the differential though a cable transmission system that also provides a secondary (2:1) gear ratio in order to satisfy the simulation derived torque requirements of the hip module, Fig. 7(b). The thigh rotation is implemented along the thigh module with the actual thigh shell forming the housing of a high power $(40 \mathrm{Nm})$ actuator group that drives this joint.

The calf section forms the housing for the two medium power actuator groups $(20 \mathrm{Nm})$ associated with knee and ankle flexion, Fig. 7(c). Torque to these joints is transferred through cable transmission systems that also provide additional secondary gearing of $(1.5: 1$ and $1.25: 1)$ for the knee and the ankle flexion joint respectively.
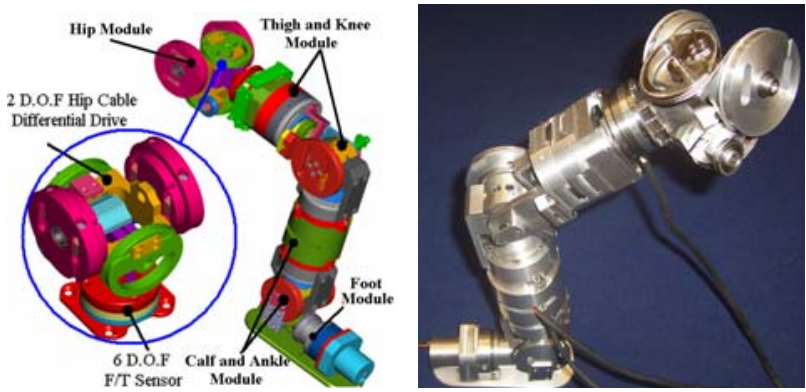

(a)



(b)
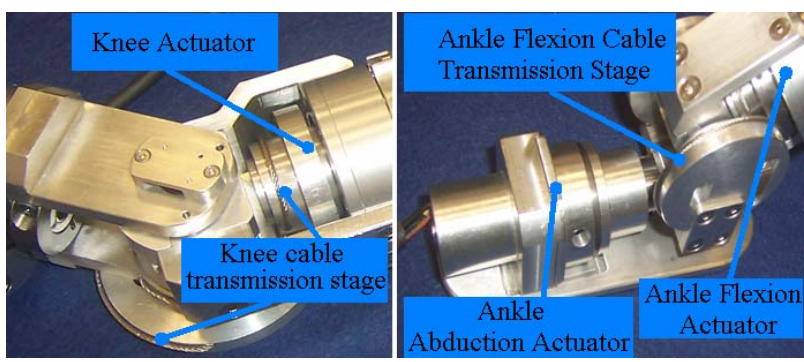

(c)

Fig. 7 CAD model and mechanical realization of the iCub leg modules.

The last D.O.F which produces ankle abduction/adduction is implemented using a low power actuator $(11 \mathrm{Nm})$ located on the foot plate and directly coupled to the ankle ab/adduction joint, Fig 8(c). The selection of actuator group for each joint was based on the joint torque/speed requirements. In joints that these conditions could be satisfied by more than one of the actuator groups mentioned in section III several iterations of the mechanical design were performed and the actuator group which optimised the design in terms of robustness, simplicity and compactness was finally selected. Fig. 8 introduces two examples of motor selection based on the simulation obtained torque/speed requirements. The two motors correspond to one of the two motors of the differential hip joint Fig. 8(a) and the knee motor, Fig. 8(b). The graphs show the torque/speed curve of the selected motors against the torque/speeds requirements of these two motors during simulated crawling motion at a cycle of $1 \mathrm{~Hz}$. It can be seen from Fig. 8 that for the selection of the actuator groups a conservative approach was adopted. This is true for most of the iCub's joints where the torque/speed margins of the actuator groups are by far exceeding the torque/speed requirements obtained by the crawling 
simulation. The main reason for this conservative selection comes from the OPEN character of the robot. As a wide range of experimentation with high level of uncertainly in terms of joint performance is the expectation from such an OPEN platform the simulation results from the crawling locomotion were only used as a guide to define the minimum performance capabilities of the $\mathrm{iCub}$ joints.

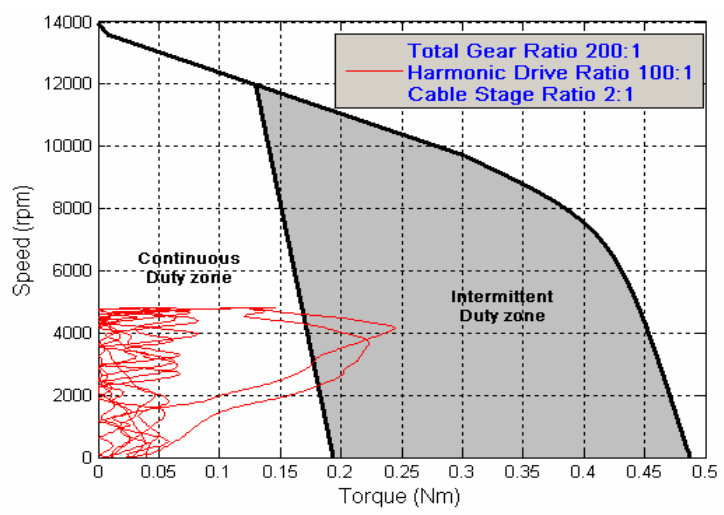

(a)



(b)

Fig. 8 Hip (a) and knee (b) motor torque speed requirements against the motor torque/speed curve.

\section{Sensing and Electronics}

Regarding the position sensing, each of the actuator units contains an incremental rotary position sensor (Hall effect sensors integrated within the stator of the frameless motor) and an absolute 12bit angular encoder as joint absolute position sensor (AS5045 from Austria Microsystems).

As far as the force/torque sensing is concerned A 6 D.O.F F/T sensor was integrated within each leg. In contrast to most of the humanoids robots where the F/T sensors are usually found at the foot level the F/T sensor in the iCub is placed at the level of the hip between the hip differential module and the thigh.

The fixation of $\mathrm{F} / \mathrm{T}$ sensor at this level of the leg is justified from the fact that during crawling the contact of the leg with the ground occurs at the knee level. In addition, force/torque sensing at the hip level enables the implementation of active compliance control at the lower limbs of the iCub. Additional F/T sensors may require though at the level of the foot for the development of the walking capability.

Both the load cell and the electronics of the F/T sensor used in $\mathrm{iCub}$ were designed in house for the purpose of dimensional optimization, Fig. 9. The 6 D.O.F load cell is based on a three spoke structure which is machined from a solid stainless steel block to reduce hysteresis and increase the strength and repeatability.

Fig. 9 also shows the developed signal conditioning and data acquisition electronics of the sensor which is based on a TMS320F2812 150MHz DSP processor. Each electronic unit with dimensions of $45[\mathrm{~mm}] \times 45[\mathrm{~mm}]$ x $5[\mathrm{~mm}]$ can handle two 6 D.O.F load cells.


Fig. 9 The structure of the 6 D.O.F force/torque sensor.

The interface between the iCub and the outside world requires only a Gbit Ethernet cable and a power cable. The robot contains the actuator power drivers, a set of DSP controllers and a $\mathrm{PC} 104$ relay station and acquisition card based on a Pentium processor. The on-board actuator control electronics for the iCub, Fig. 10, are embedded at or near the motor/ joint assemblies and are primarily responsible for the monitoring of the actuator sensory signals and the generation of the control signals.

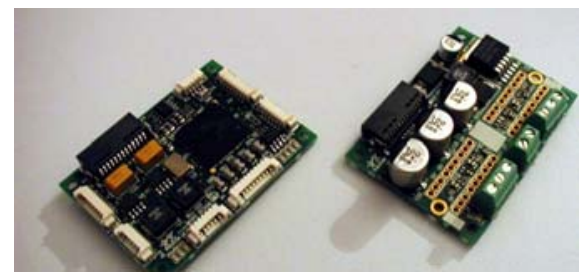

Fig. 10: The motor control and driver boards.

\section{LOWER BODY ACTUATION CHARACTERISTIC MEASURES}

The motion range and torque measure of the first prototype of the individual joints of iCub lower body are introduced in Table IV. As can be seen in this table and as far as the torque requirements of the $\mathrm{iCub}$ are concerned these are fully satisfied by the proposed design and actuator selection. In some joints significantly higher torques are achieved. Regarding the range of motion of the individual joints these in general also meet the specified requirements of the iCub with some small limitations in the hip and knee flexion ranges. These are currently being addressed in the first revision of the design and are considered not significant issues. 
TABLE IV

CHARACTERISTIC MEASURES OF THE ICUB.

\begin{tabular}{|c|c|c|c|c|c|}
\hline Leg & $\begin{array}{c}\text { Range of } \\
\text { motion }\left({ }^{\circ}\right)\end{array}$ & $\begin{array}{c}\text { Actuator } \\
\text { (Kollmorgen } \\
\text { series) }\end{array}$ & Gear & $\begin{array}{c}\text { Secondary } \\
\text { Cable } \\
\text { Gearing }\end{array}$ & Torque (Nm) \\
\hline Hip Flex/Ext & $+50,-100$ & RBE 1210 & HD-CSG14_100:1 & $2: 1$ & Sum of the differential \\
drive torques=84Nm
\end{tabular}

\section{CONCLUSIONS}

This paper discussed the concepts adopted for the design and fabrication of the lower body of the OPEN embodied robotic child (iCub). The development of such an OPEN structure posed many significant challenges and required careful design and integration of the various components to ensure robustness under a wide range of experimental conditions which are highly probable.

To ease manufacturing and maintenance a modular approach was followed during the design process. Highly integrated actuator modules were developed to power the individual joints of the iCub's lower body. The minimum number of different actuator groups used eases the fabrication procedure and is also beneficial from the maintenance point of view. Data obtained from crawling locomotion simulations were used to define the torque speed requirements of the individual joints. To ensure robustness under wide experimentation with high level of uncertainly a conservative approach was adopted during the actuation selection process with the simulation data being used to set the minimum specifications for the actuator groups. Performance measures depict the ability of the first prototype to meet the $\mathrm{iCub}$ simulation torque and motion requirements.

\section{ACKNOWLEDGMENT}

This work is supported by the European Commission FP6, Project IST-004370

\section{REFERENCES}

[1] J. Yamaguchi, E. Soga, S. Inoue, and A. Takanishi, "Development of a Bipedal Humanoid Robot - Control Method of Whole Body Cooperative Dynamic Biped Walking," Proc. IEEE ICRA 1999, pp. 368-374.

[2] K. Nishiwaki, T. Sugihara, S. Kagami, F. Kanehiro, M. Inaba, and H. Inoue, "Design and Development of Research Platform for Perception-Action Integration in Humanoid Robot: H6," Proc. IEEE/RSJ IROS 2000, pp. 1559-1564.

[3] K. Hirai, M. Hirose, Y. Haikawa, and T. Takenaka, "The Development of Honda Humanoid Robot," Proc. IEEE ICRA 1998, pp. 1321-1326.
[4] M. Hirose, Y. Haikawa, T. Takenaka, and K, Hirai, "Development of Humanoid Robot ASIMO," Proc. IEEE/RSJ IROS 2001, Workshop2 .

[5] S. Lohmeier, T. Buschmann, H. Ulbrich, F. Pfeiffer, "Modular Joint Design for Performance Enhanced Humanoid Robot LOLA", Proc. of IEEE ICRA 2006, pp 88-93, Orlando, Florida.

[6] Yu Ogura, Hiroyuki Aikawa, Kazushi, Shimomura, Hideki Kondo, and Akitoshi Morishima, Hun-ok Lim Atsuo Takanishi, "Development of a New Humanoid Robot WABIAN-2", Proc. of IEEE ICRA 2006, pp 76-81, Orlando, Florida.

[7] J.-Y. Kim, I.-W. Park, J. Lee, M.-S. Kim, B.-K. Cho, and J.-H. Oh, "System design and dynamic walking of humanoid robot KHR-2," in Proc. of IEEE ICRA 2005, pp. 1443-1448.

[8] Bram Vanderborght, Bj"orn Verrelst, Ronald Van Ham, Micha"el Van Damme, Pieter Beyl and Dirk Lefeber, "Torque and Compliance Control of the Pneumatic Artificial Muscles in the Biped "Lucy", Proc of IEEE ICRA 2006, pp 842-847, Orlando, Florida.

[9] K. Kaneko , F. Kanehiro, S. Kajita, H. Hirukawa, T.Kavasaki, M. Hirata, K. Akachi, and T. Isozumi, "Humanoid Robot HRP-2", Proc. IEEE ICRA 2004, New Orleans.

[10] Satoru Shirata, Atsushi Konno, and Masaru Uchiyama,"Design and Development of a Light-Weight Biped Humanoid Robot Saika-4", Proc. IEEE/RSJ IROS 2004, pp148-153, Sendai, Japan,

[11] I. Mizuuchi, R.Tajima, T.Yoshikai, D.Sato, K.Nagashima, M Inaba, Y.Kuniyoshi and H.Inoue, " The Design and Control of the Flexible Spine of a Fully Tendon-Driven Humanoid "Kenta", Proc. IEEE/RSJ IROS 2002, pp2527-2532, Lausanne, Swizerland.

[12] T. Furuta, Y. Okomura, and K. Tomiyama, "Design and Construction of a Series of Compact Humanoid Robots and Development of Biped Walk Control Strategies,” Proc. IEEE-RAS Int. Conf on Humanoid Robots, 2000.

[13] F. Yamasaki, T. Matsui, T. Miyashita, and H. Kitano, "PINO The Humanoid that Walks", Proc. IEEE-RAS Int. Conference on Humanoid Robots,2000.

[14] Y. Kuroki, T. Ishida, J. Yamaguchi, M. Fujita, and T. Doi, “A Small Biped Entertainment Robot," Proc. IEEE-RAS Int.Conference on Humanoid Robots, pp. 181-186, 2001

[15] W.G.Wu, et al, "Design, Simulation and Walking Experiment for a Humanoid and Gorilla Robot with Multiple Locomotion Modes", Proc. of EEE/RSJ IROS 2005, pp.44-49.

[16] G. Sandini, G. Metta, and D Vernon, “ RobotCub: An Open Framework for Research in Embodied Cognition", International Journal of Humanoid Robotics, November 18, 2004 8:2

[17] Ikuo Mizuuchi, Ryosuke Tajima, Tomoaki Yoshikai, Daisuke Sato, Koichi Nagashima, Masayuki Inaba, Yasuo Kuniyoshi, and Hirochika Inoue: "The Design and Control of the Flexible Spine of a Fully Tendon-Driven Humanoid Kenta", Proc. IEEE/RSJ IROS 2002, pp.2527--2532, Lausanne, Switzerland.

[18] O. Michel, "Webots: Professional mobile robot simulation," International Journal of Advanced Robotic Systems, vol. 1, no. 1, pp.39-42, 2004 . 